\section{Tendências temporais de atividade física e comportamento sedentário em Porto Alegre, Brasil: 2006-2012}

Time trends of physical activity and sedentary behavior in Porto Alegre, Brazil: 2006-2012

\author{
Alex Sander Souza de Souza' \\ Shana Ginar da Silva² \\ Grégore Iven Mielke ${ }^{2}$ \\ Pedro Curi Hallal ${ }^{1,2}$
}

\section{RESUMO}

O objetivo do estudo é analisar tendências temporais de atividade física e comportamento sedentário e sua relação com indicadores sociodemográficos, em adultos da cidade de Porto Alegre, RS, Brasil, no período de 2006 a 2012. Foram analisados dados de 13,721 adultos (18+ anos) participantes do Sistema de Vigilância de Fatores de Risco e Proteção para Doenças Crônicas por Inquérito Telefônico (VIGITEL). Tendências temporais foram analisadas para os indicadores: atividade física no lazer ( $\geq 5$ dias/semana; $\geq 30$ minutos/ dia), atividade física no deslocamento ( $\geq 30$ minutos/dia caminhando ou pedalando para ir/ voltar do trabalho) e comportamento sedentário ( $>3$ horas/dia assistindo televisão). A atividade física de lazer se manteve estável ao longo dos sete anos, havendo um leve aumento de 3,5\% ( $p=0,03)$ de 2006 para 2012 entre os homens, enquanto nas mulheres, no mesmo período, o índice baixou de $13,3 \%$ para $11,5 \%(\mathrm{p}=0,03)$. Já em relação à atividade física no deslocamento, o percentual de ativos variou de 10,4\% em 2006 para 10,1\% em 2008 (p= 0,95). Entre 2009 e 2012, o percentual baixou de $12,2 \%$ para $10,2 \%$ ( $\mathrm{p}=0,05)$. $\mathrm{Na}$ análise do comportamento sedentário, observou-se um declínio de $28,9 \%$ para $24,9 \%(\mathrm{p}=0,03)$ de 2006 para 2009 , e de $27,3 \%$ para $25,6 \%$ ( $p=0,21)$ entre 2010 e 2012 . Os dados do presente estudo mostram que a atividade física de lazer está estável na amostra total, mas aumentou levemente entre os homens e diminuiu levemente entre as mulheres. Já a atividade física no deslocamento e o comportamento sedentário mostraram períodos de quedas e estabilização.

\section{PALAVRAS-CHAVE}

Atividade motora; Vigilância em saúde pública; Vigilância populacional; Epidemiologia.

\begin{abstract}
The aim of the present study was to evaluate time trends of physical activity and sedentary time from 2006 to 2012 and their associations with sociodemographic indicators among adults living in Porto Alegre, Brazil. We analyzed data from 13,721 adults (18+ years) participants of the Surveillance System on Risk and Protective Factors for Chronic Disease through Telephone Interviews (VIGITEL). Time trends of the following indicators were evaluated: leisure-time physical activity ( $\geq 5$ days/week; $\geq 30$ min/day), commuting physical activity $\geq 30$ min/day walking or cycling to/from work) and sedentary time (>3 hours per day watching TV). Leisure-time physical activity remained stable in the seven years period, with a slight increase of $3.5 \%(P=0.03)$ among men and a slight decrease from $13.3 \%$ to $11.5 \%(P=0.03)$ among women. The proportion of individuals classified as active in commuting ranged from $10.4 \%$ in 2006 to $10.1 \%$ in $2008(P=0.95)$ and then from $12.2 \%$ to $10.2 \%$ from 2009 to $2012(P=0.05)$. Sedentary time decreased from $28.9 \%$ in 2006 to $24.9 \%$ in $2009(P=0.03)$ and from 27.3\% in 2010 to 25.6\% in $2012(P=0.21)$. Data from the present study show that leisure-time physical activity is stable in Porto Alegre when both sexes are analyzed together. Commuting physical activity and sedentary time showed periods of decline and stabilization.
\end{abstract}

\section{KEYWORDS}

Motor activity; Public health surveillance; Population surveillance; Epidemiology.
Rev Bras Ativ Fis Saúde p. 514-522 DOI

http://dx.doi.org/10.12820/rbafs.v.19n4p514

1 Programa de Pós-graduação em Educação Física, Universidade Federal de Pelotas

2 Programa de Pós-graduação em Epidemiologia, Universidade Federal de Pelotas 


\section{INTRODUCCÃO}

Nas últimas décadas, ocorreram mudanças marcantes nos modos de transporte e houve incremento da inserção da tecnologia nas atividades de lazer ${ }^{1}$. Este novo cenário resultou em alterações no perfil de morbimortalidade da população, hoje caracterizado pela predominância das doenças crônicas não transmissíveis (DCNT's) ${ }^{2}$. Nesse contexto, a inatividade física e o comportamento sedentário são fatores de risco importantes à saúde da população ${ }^{3}$. Entre as consequências do sedentarismo está o risco aumentado de complicações cardiovasculares e metabólicas ${ }^{3}$. Já a inatividade física aumenta o risco de diversas DCNT's, desordens mentais e morte prematura, causando 5,3 milhões de mortes por ano no mundo ${ }^{4,5}$.

A prevalência global de inatividade física em adultos é de $31 \%$, variando de $17 \%$ na Ásia a $43 \%$ nas Américas ${ }^{6}$. O percentual de adultos no mundo que passam quatro ou mais horas na frente da televisão é de $42 \%{ }^{6}$. No entanto, grande parte das informações disponíveis sobre esses comportamentos são oriundas de inquéritos pontuais, havendo uma carência de estudos de tendências temporais.

Os estudos de tendência, em sua maioria, avaliam especificamente a atividade física de lazer ${ }^{7-10}$. Ampliar o enfoque é fundamental, visto que os outros domínios têm relevância na atividade física total, principalmente em países de renda média ou baixa, como o Brasil ${ }^{11}$. $\mathrm{O}$ monitoramento dos fatores de riscos para DCNT's, entre eles a inatividade física, é importante na criação de políticas públicas. Estudos prévios relatam que os quatro domínios da atividade física (ocupacional, lazer, deslocamento, serviços domésticos) estão fortemente relacionados ao gênero, idade, escolaridade e nível social ${ }^{11,12}$. Pesquisas anteriores sugerem que homens jovens, de classe social mais alta e com maior escolaridade são mais ativos no lazer ${ }^{8}$. No entanto, quando o objeto de estudo é a atividade física no deslocamento, a atividade física é mais frequente em indivíduos com grau de escolaridade baixo ${ }^{11}$.

No Brasil, o Sistema de Vigilância de Fatores de Risco e Proteção para Doenças Crônicas por Inquérito Telefônico (VIGITEL) faz o monitoramento dos principais fatores de risco e proteção para as DCNT's em todas as capitais brasileiras e no Distrito Federal ${ }^{13}$. Análises nacionais já foram conduzidas $^{14}$, mas pouca ênfase tem sido dada a investigações mais detalhadas sobre as tendências de atividade física em cada capital brasileira. Tais informações são essenciais para um planejamento local de promoção da saúde, com ênfase na promoção da atividade física. Este estudo tem como objetivo analisar a tendência temporal de atividade física e do comportamento sedentário e sua relação com indicadores sociodemográficos, em adultos da cidade de Porto Alegre, RS, Brasil, no período de 2006 a 2012.

\section{MÉTODOS}

As análises apresentadas neste estudo são baseadas nos dados do VIGITEL dos anos de 2006 a 2012. O VIGITEL é um inquérito telefônico de base populacional realizado, desde 2006, pelo Ministério da Saúde nas 26 capitais brasileiras e no Distrito Federal. A população inclui adultos ( $\geq 18$ anos) que possuem linha de telefone fixa em seus domicílios. 
Em cada capital, a amostragem foi realizada em duas etapas: (1) seleção randômica das linhas telefônicas residenciais fixas e (2) seleção aleatória do morador do domicílio que será entrevistado. Foram entrevistadas aproximadamente 2.000 pessoas em cada capital e no Distrito Federal, de modo a garantir um erro máximo de cerca de dois pontos percentuais para as estimativas de prevalência de qualquer fator de risco analisado ${ }^{13}$. Para o presente estudo, foram utilizados sete inquéritos da capital do estado do Rio Grande do Sul, Porto Alegre. Mais informações sobre os procedimentos amostrais do VIGITEL estão disponíveis em outras publicações ${ }^{13}$.

A análise da prática de atividade física considerou dois domínios: lazer e deslocamento. No lazer, a prática de atividade física foi mensurada com base em questões sobre a prática de exercício físico ou esporte nos últimos três meses e, em caso positivo, duração e frequência semanal dessa prática. O domínio do deslocamento foi avaliado através de questões sobre o uso de caminhada ou bicicleta para ir ou voltar do trabalho, sendo também computadas a duração e frequência semanal dessa prática, em casos positivos. Outro fator analisado pelo VIGITEL é o comportamento sedentário, avaliado pelo tempo diário despendido assistindo televisão.

Os desfechos foram categorizados da seguinte maneira: (a) ativos no lazer: praticar pelo menos 30 minutos por dia de esportes em pelo menos cinco dias da semana; (b) ativos no deslocamento: fazem uso de caminhada ou bicicleta para ir e/ou voltar do trabalho por pelo menos 30 minutos por dia; (c) comportamento sedentário: relatar mais de três horas por dia assistindo televisão. Os indicadores propostos são semelhantes aos indicadores utilizados em publicação prévia ${ }^{14}$.

Em análises adicionais, para uma melhor compreensão da distribuição da prática da atividade física no lazer, a variável também foi analisada em quatro categorias: (a) indivíduos que não fazem nenhuma atividade física no lazer; (b) indivíduos que fazem alguma atividade física no lazer, mas por menos de três vezes na semana e menos de 20 minutos por dia; (c) indivíduos que praticam atividade física no lazer pelo menos três vezes por semana durante 20 minutos, mas praticam menos de cinco dias por semana ou menos de 30 minutos por dia; (d) indivíduos que praticam atividade física no lazer pelo menos cinco vezes por semana durante pelo menos 30 minutos por dia.

As questões do domínio de deslocamento sofreram modificações no ano de 2009. Foram adicionadas informações referentes ao deslocamento para a escola e/ou universidade. Desse modo, para a análise de tendência, foram considerados dois períodos: 2006 a 2008 e 2009 a 2012. Do mesmo modo, as questões que mensuravam o tempo despendido assistindo televisão sofreram modificações em 2010. Logo, para as análises de tendências, foram considerados dois períodos: 2006 a 2009 e 2010 a 2012. Para fins de análise, pesos pós-estratificados foram utilizados para dar conta da amostragem, de modo que os resultados pudessem ser extrapolados para a população das capitais. O método Rake foi usado para estimar a população de cada ano com base em dados do censo ${ }^{15,16}$.

As tendências temporais para cada indicador foram estratificadas por sexo (masculino; feminino), idade (18-24; 25-34; 35-44; 45-54; 55-64; 65+ anos) e escolaridade $(0-4,5-8,9-11,12+$ anos). O percentual de mudança ao longo do tempo e a significância estatística das mudanças foi calculada pela regressão de 
Poisson, tendo os indicadores de atividade física e comportamento sedentário como desfecho de interesse e o ano de pesquisa como a variável de exposição. As análises foram realizadas utilizando o pacote estatístico Stata versão 12.1 (Stata Cort., College Station, EUA).

O VIGITEL foi aprovado pela Comissão de Ética em Pesquisa em Seres Humanos do Ministério da Saúde. Por se tratar de entrevista por telefone, o termo de consentimento livre e esclarecido foi substituído pelo consentimento verbal obtido por ocasião dos contatos telefônicos com os entrevistados.

\section{RESULTADOS}

A Tabela 1 mostra o número de linhas elegíveis, entrevistas, taxa de sucesso e características sociodemográficas da amostra. A taxa de sucesso do VIGITEL variou entre 2006 e 2012, sendo o menor índice observado no ano de 2011 $(56,2 \%)$ e o maior em 2009 (78,7\%). O tamanho da amostra tem se mantido uniforme ao longo dos anos, com exceção de 2012, onde por questões financeiras o número de entrevistas foi $20 \%$ menor. No total, as análises consideram dados de 13.721 indivíduos. A idade média e os anos de escolaridade têm tido um leve aumento ao decorrer dos anos.

TABELA 1 - Linhas elegíveis, número de entrevistas, tamanho amostral e características sociodemográficas das amostras do VIGITEL, 2006 - 2012.

\begin{tabular}{lccccccc}
\hline Indicadores & 2006 & 2007 & 2008 & 2009 & 2010 & 2011 & 2012 \\
\hline Linhas elegíveis & 3.120 & 3.128 & 2.829 & 2.553 & 2.563 & 3.553 & 2.677 \\
\hline $\begin{array}{l}\text { Número de } \\
\text { entrevistas }\end{array}$ & 2.010 & 2.002 & 2.013 & 2.010 & 2.005 & 2.000 & 1.665 \\
\hline Taxa de sucesso (\%) & 64,42 & 64,00 & 71,15 & 78,73 & 78,22 & 56,29 & 62,19 \\
\hline Tamanho de amostra & 2.010 & 2.002 & 2.013 & 2.010 & 2.005 & 2.016 & 1.665 \\
\hline$\%$ mulheres & 54,89 & 54,90 & 54,90 & 54,90 & 54,91 & 54,91 & 54,92 \\
\hline Idade média & 46.74 & 46.60 & 47.77 & 48.80 & 49.16 & 51.18 & 51.78 \\
\hline Escolaridade média & 10.82 & 11.92 & 11.43 & 11.68 & 11.28 & 10.64 & 11.10 \\
\hline
\end{tabular}

A Figura 1 apresenta as tendências temporais de atividade física e do comportamento sedentário em adultos de Porto Alegre no período de 2006 a 2012. A prevalência de ativos no lazer passou de 13,8\% no ano de 2006 para $14,4 \%(p=0,65)$ no ano de 2012. A variação de ativos no deslocamento entre os anos de 2006 e 2008 foi de 10,4\% para 10,1\% (p=0,95). Já entre 2009 e 2012, o percentual baixou de $12,2 \%$ para $10,2 \%(\mathrm{p}=0,05)$. O hábito de assistir TV diminuiu de $28,9 \%$ a 24,9\% ( $\mathrm{p}=0,03)$ de 2006 para 2009. Já no período de 2010 a 2012, a variação foi de $27,3 \%$ para $25,6 \%(p=0,21)$.

A Figura 2 mostra a distribuição da prática de atividade física no lazer de acordo com quatro categorias. $\mathrm{O}$ percentual de indivíduos que não relata qualquer prática de atividade física no lazer permaneceu estável no período, assim como o percentual de indivíduos com prática de pelo menos cinco dias por semana, pelo menos 30 minutos por dia. Em 2012, 51,1\% dos indivíduos não realizavam qualquer atividade física no lazer e apenas $13,1 \%$ praticavam $5 \times 30$ de minutos ou mais. 


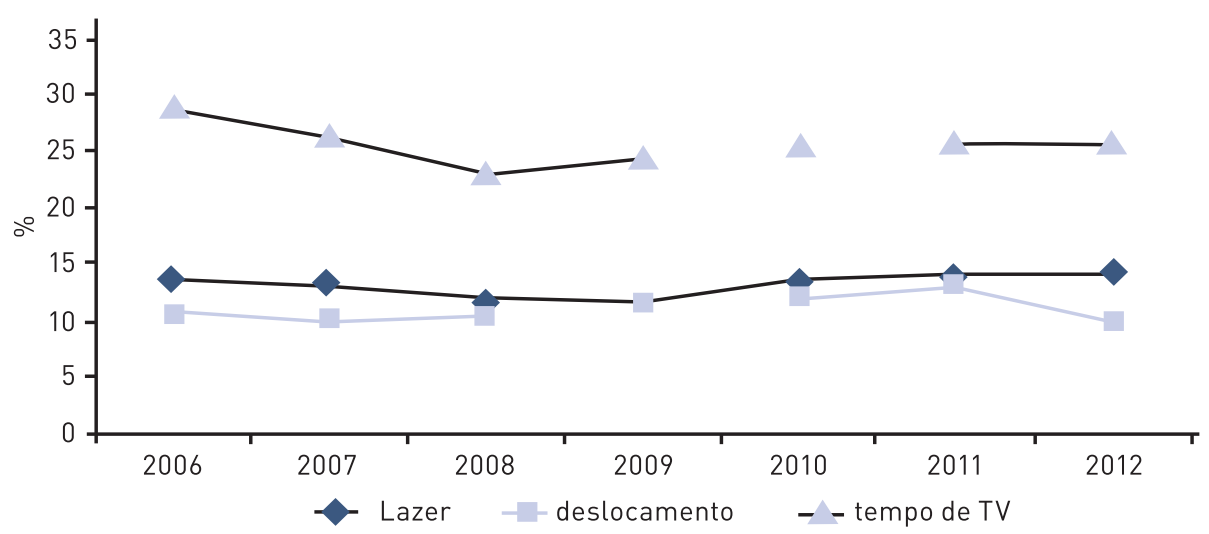

FIGURA 1 - Prevalência de ativos no lazer e no deslocamento e tempo de TV, entre o período de 2006 a 2012, Porto Alegre, Brasil.

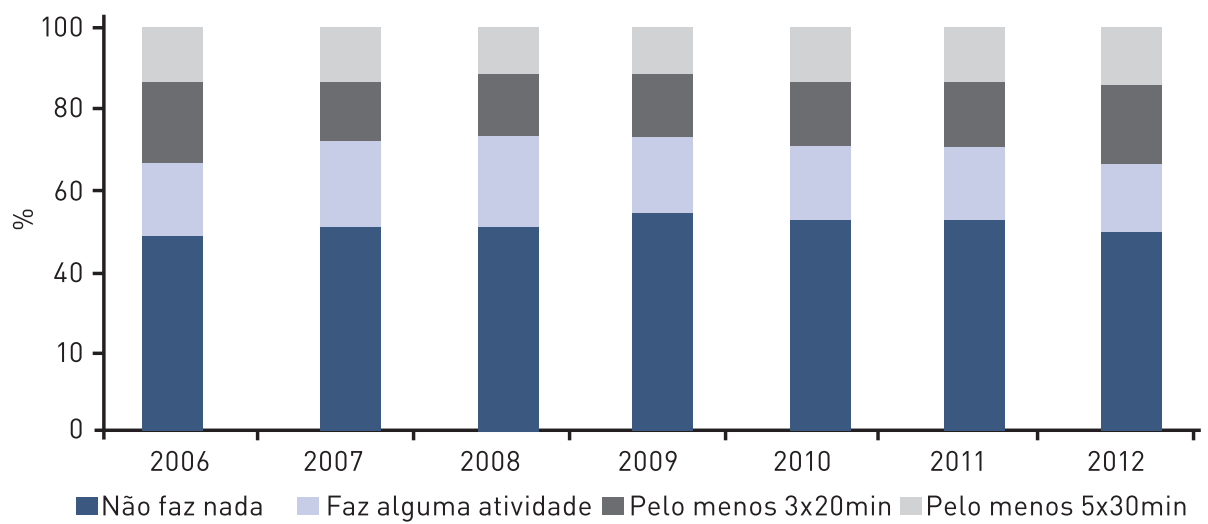

FIGURA 2 - Distribuição da prática de atividade física no lazer, no período de 2006 a 2012, Porto Alegre, Brasil.

A prevalência de ativos no lazer (Tabela 2) foi significativamente maior nos homens em todos os anos, exceto 2007. Nos homens houve um aumento de 3,5\% ( $p=0,03)$ de 2006 para 2012, enquanto nas mulheres, no mesmo período, o índice baixou de $13,3 \%$ para $11,5 \%(\mathrm{p}=0,03)$. O percentual de ativos não variou significativamente em nenhum grupo etário, embora um aumento de 4,2\% tenha sido observado de 2006 a 2012 entre os mais jovens (18-24 anos) e um decréscimo de 1,7\% tenha sido detectado entre aqueles de 25 a 34 anos. Da mesma forma, o percentual de ativos no lazer não variou significativamente conforme a escolaridade dos entrevistados, embora tenha se identificado um aumento de 4,1\% naqueles com menor escolaridade (0-4 anos) e um decréscimo de $3,4 \%$ naqueles com maior escolarização (12+ anos).

O percentual de ativos no deslocamento (Tabela 3) foi maior nos homens do que nas mulheres, nos dois períodos de comparação (2006 a 2008 e 2009 a 2012). A prevalência entre os homens foi de 14,1\% em 2006 passando para $12,8 \%$ em 2008 ( $\mathrm{p}=0,91$ ). Já entre 2009 e 2012, os índices variaram de 12,4\% para $10,9 \%(p=0,15)$. Entre as mulheres, os percentuais entre 2006 e 2008 foram respectivamente de $7,4 \%$ e $7,9 \%(\mathrm{p}=0,78)$. Já entre 2009 e 2012, a prevalência de mulheres ativas variou de $10,9 \%$ e $9,6 \%(\mathrm{p}=0,48)$, respectivamente. A variação da prevalência de ativos no deslocamento foi pequena, sem qualquer diferença estatisticamente significativa. Em relação à escolaridade, houve aumento significativo na prevalência de ativos no deslocamento entre aqueles com 9 a 11 anos de estudo, no primeiro período analisado. 
TABELA 2 - Prevalência de ativos no lazer estratificados por sexo, idade e escolaridade.

\begin{tabular}{lccccccccc}
\hline Variáveis & 2006 & 2007 & 2008 & 2009 & 2010 & 2011 & 2012 & $\begin{array}{c}\Delta \text { de } \\
\text { Variação \% }\end{array}$ & $p$ \\
\hline Sexo & & & & & & & & & \\
\hline Homens & 14,2 & 13,1 & 14,3 & 14,8 & 17,3 & 16,6 & 17,8 & $+3,6$ & 0,03 \\
\hline Mulheres & 13,3 & 13,4 & 9,3 & 8,7 & 10,4 & 11,7 & 11,5 & $-3,2$ & 0,03 \\
\hline Faixa etária & & & & & & & & & \\
\hline 18-24 & 15,6 & 13,6 & 15,2 & 17,5 & 23,4 & 18,9 & 19,7 & $+3,9$ & 0,17 \\
\hline 25- 34 & 15,1 & 11,5 & 10,1 & 10,6 & 13,9 & 12,0 & 13,4 & $-3,3$ & 0,36 \\
\hline 35- 44 & 11,6 & 12,1 & 9,9 & 5,2 & 11,0 & 13,4 & 15,0 & $+3,4$ & 0,28 \\
\hline 45- 54 & 12,0 & 10,5 & 11,2 & 8,6 & 9,9 & 12,7 & 10,9 & $-3,4$ & 0,18 \\
\hline 55- 64 & 14,5 & 19,2 & 10,5 & 10,9 & 11,2 & 15,2 & 14,3 & $-2,9$ & 0,22 \\
\hline 65 ou + & 13,7 & 15,6 & 13,8 & 18,7 & 12,3 & 13,0 & 14,1 & $+1,3$ & 0,18 \\
\hline Escolaridade & & & & & & & & & \\
\hline 0- 4 anos & 10,3 & 12,9 & 12,3 & 11,4 & 5,1 & 8,6 & 14,8 & $+1,1$ & 0,73 \\
\hline 5- 8 anos & 12,9 & 10,2 & 7,8 & 7,2 & 11,8 & 10,6 & 14,3 & $+1,0$ & 0,73 \\
\hline 9- 11 anos & 14,7 & 14,7 & 13,3 & 12,1 & 16,9 & 16,4 & 16,2 & $+0,3$ & 0,85 \\
\hline 12 ou + anos & 15,2 & 13,9 & 12,4 & 14,1 & 14,2 & 15,5 & 11,8 & $-1,4$ & 0,43 \\
\hline
\end{tabular}

TABELA 3 - Prevalência de ativos no deslocamento estratificado por sexo, idade e escolaridade.

\begin{tabular}{|c|c|c|c|c|c|c|c|c|c|c|c|}
\hline Variáveis & 2006 & 2007 & 2008 & $\Delta$ de variação & $p$ & 2009 & 2010 & 2011 & 2012 & $\Delta$ de variação & $\mathrm{p}$ \\
\hline \multicolumn{12}{|l|}{ Sexo } \\
\hline Homens & 14,1 & 10,5 & 12,8 & $-0,8$ & 0,91 & 12,4 & 13,5 & 15,6 & 10,9 & $-3,0$ & 0,15 \\
\hline Mulheres & 7,4 & 9,6 & 7,9 & $+2,2$ & 0,78 & 10,9 & 11,2 & 11,2 & 9,6 & $-7,4$ & 0,48 \\
\hline \multicolumn{12}{|l|}{ Faixa etária } \\
\hline $18-24$ & 13,1 & 13,6 & 10,1 & $-17,8$ & 0,14 & 15,3 & 12,7 & 15,9 & 9,2 & $-11,2$ & 0,50 \\
\hline $25-34$ & 15,1 & 12,4 & 16,1 & $+7,8$ & 0,44 & 16,3 & 15,3 & 17,3 & 11,5 & $-3,7$ & 0,85 \\
\hline $35-44$ & 10,9 & 12,7 & 12,1 & $+1,3$ & 0,89 & 12,2 & 17,2 & 15,2 & 12,6 & $+1,2$ & 0,95 \\
\hline 45- 54 & 10,9 & 10,5 & 9,2 & $-8,1$ & 0,48 & 11,2 & 12,9 & 13,7 & 13,7 & $+1,0$ & 0,85 \\
\hline $55-64$ & 6,6 & 5,4 & 7,0 & $+0,4$ & 0,98 & 9,6 & 6,7 & 10,1 & 7,7 & $-5,6$ & 0,31 \\
\hline $65 \mathrm{ou}+$ & 1,4 & 1,4 & 1,5 & $+11,6$ & 0,59 & 1,3 & 4,7 & 3,4 & 4,0 & $+20,0$ & 0,91 \\
\hline \multicolumn{12}{|l|}{ Escolaridade } \\
\hline $0-4$ anos & 7,4 & 10,7 & 9,2 & $+5,9$ & 0,77 & 7,6 & 12,1 & 13,9 & 7,9 & $-0,6$ & 0,51 \\
\hline 5- 8 anos & 14,6 & 12,4 & 8,8 & $-18,3$ & 0,12 & 14,9 & 13,1 & 13,7 & 15,4 & $-1,4$ & 0,48 \\
\hline 9- 11 anos & 9,6 & 9,8 & 12,6 & $+2,0$ & 0,03 & 12,6 & 12,0 & 14,7 & 10,2 & $-7,1$ & 0,65 \\
\hline 12 ou + anos & 10,1 & 7,9 & 9,0 & $-11,2$ & 0,18 & 9,7 & 11,8 & 10,7 & 7,4 & $-3,3$ & 0,25 \\
\hline
\end{tabular}

\section{DISCUSSÃO}

Os níveis de atividade física no lazer se mostraram estáveis em Porto Alegre no período de 2006 a 2012, assim como no deslocamento no período de 2006 a 2008. Já no período de 2009 a 2012, observou-se uma diminuição na prevalência de ativos no deslocamento. O comportamento sedentário, expresso pelo tempo assistindo televisão, obteve um decréscimo entre os anos de 2006 a 2009, enquanto que entre os anos de 2010 a 2012 manteve-se estável. Este é um dos primeiros estudos de tendência temporal da atividade física que 
analisou mais de um domínio e ainda o comportamento sedentário em uma amostra de adultos no Brasil. Os resultados do presente estudo são de grande importância para o monitoramento dos níveis de atividade física no Brasil e para a compreensão do comportamento dos indivíduos nos diferentes domínios segundo indicadores sociodemográficos.

Algumas limitações da presente análise devem ser levadas em consideração. Primeiramente, o VIGITEL é restrito para indivíduos que possuem linha telefônica fixa. Cabe destacar, no entanto, que Porto Alegre é uma das capitais brasileiras com maior percentual de cobertura de telefonia. Uma restrição importante é a mensuração do comportamento sedentário, pois o VIGITEL utiliza apenas o número de horas assistindo televisão como indicativo do comportamento sedentário, o que pode não refletir bem o padrão de comportamento sedentário de uma população ${ }^{17}$.

Dados sobre tendências temporais de atividade física estão disponíveis na cidade de Pelotas, no interior do Rio Grande do Sul. Houve um grande aumento da inatividade física (considerando os quatro domínios) entre 2002 e 2007, e uma relativa estabilização entre 2007 e 2012 $2^{23}$. Especificamente no tempo livre, o estudo mostrou uma estabilização dos níveis de atividade física entre 2003 e 2010, consistente com os achados do presente estudo ${ }^{18}$. Desta forma, é provável que o declínio na atividade física total, observado no estudo de Hallal e colegas sejam em decorrência de diminuição na prática em outros domínios.

Estudo realizado no estado de São Paulo entre 2002 e 2008, mostrou uma queda na prevalência de inatividade física nos quatro domínios analisados conjuntamente ${ }^{19}$. No entanto, dados desse estudo devem ser interpretados com cautela, visto que o estado está exposto a um programa de intervenção para promoção da atividade física.

Utilizando dados de todas as capitais, o estudo de Mielke e colaboradores relatou um leve aumento nos níveis de atividade física no lazer entre 2006 e 2012, resultado similar ao observado no presente estudo entre os homens ${ }^{14}$. Em relação à atividade física de deslocamento, tanto o estudo de Mielke et al. quanto o presente estudo encontraram declínio ${ }^{14}$. Em relação ao comportamento sedentário, o estudo nacional encontrou leve declínio entre 2006 e 2009 na prevalência de sujeitos que assistiam três ou mais horas de televisão por dia $^{14}$. Em todas as comparações entre nosso estudo e a análise dos dados nacionais é fundamental levar em consideração que o poder estatístico do estudo nacional é bastante superior ao de nosso estudo, tendo em vista o tamanho de amostra total.

Outra consideração a ser feita na comparação entre os estudos disponíveis na literatura nacional é que os inquéritos de Pelotas ${ }^{18}$ e São Paulo ${ }^{19}$ utilizaram o IPAQ, enquanto outros estudos, inclusive o nosso, utilizaram o instrumento do VIGITEL. Embora apresentem algumas similaridades, os instrumentos não são comparáveis e inviabilizam a comparação das estimativas pontuais.

A maioria dos estudos de tendência temporal são de países de renda alta. A literatura internacional mostra um leve aumento nas atividades físicas de lazer ${ }^{8,20}$ diferente dos achados do presente estudo. Na atividade física no deslocamento, os resultados não são uniformes. Em um estudo realizado no Canadá ${ }^{12}$, homens e mulheres tornaram-se mais ativos no deslocamento com o passar do tempo. Já na Catalunha, na Espanha, o nível de ativos no transporte se manteve estável entre os homens, e diminui entre as mulheres, em um perí- 
odo de 11 anos $^{20}$, semelhante a estudo realizado na China ${ }^{21}$, onde os níveis de atividade física no deslocamento não se alteraram no período de 1991 a 2011.

A queda no índice de ativos no deslocamento provavelmente reflete a falta de estrutura para o deslocamento ativo, a falta de planejamento urbano, a carência de vias públicas adequadas para a caminhada e/ ou o ciclismo e as dificuldades para uma melhor organização do trânsito. Outro fator que pode justificar a queda no índice de ativos no transporte é o fato de haver maior poder aquisitivo entre as famílias, facilitando a aquisição de transportes motorizados ${ }^{21}$.

Em relação à atividade física realizada no lazer, a mesma está estável no período de análise do presente estudo. Algumas hipóteses para este resultado podem ser a falta de segurança, a restrição ao acesso de locais privados que proporcionam atividades de lazer, bem como ciclovias, trilhas e áreas verdes adequadas aos esportes e com boa qualidade ${ }^{18}$. A diferença entre homens e mulheres em relação à prática de atividade física de lazer, e atividade física no deslocamento é similar aos resultados de estudos prévios ${ }^{18,22,23}$, onde há o relato de homens serem mais ativos que as mulheres.

Analisar as tendências temporais da atividade física é importante para a saúde pública do Brasil, visto que dados prévios são quase que exclusivos de países de renda alta. Tais dados permitem o planejamento governamental na construção de sociedades nas quais a escolha de ser fisicamente ativo não seja apenas vista como saudável, mas também seja segura, acessível e valorizada pela população ${ }^{6}$. A continuidade do monitoramento dos fatores de risco pelo VIGITEL é importante e necessária, havendo assim a possibilidade de futuras avaliações de tendências temporais para vários indicadores, entre eles a prática de atividade física.

\section{REFERÊNCIAS}

1. Alves US. Não ao sedentarismo, sim à saúde: contribuição da educação física escolar e dos esportes. O Mundo da Saúde São Paulo. 2007; 31(4): 464- 9.

2. Schmidt MN, Duncan BB, Silva GA, Menezes AM, Monteiro CA, Barreto SM, et al. Chronic non-communicable diseases in Brazil: burden and current challenges. Lancet. 2012; 377: 1949- 61.

3. Tremblay, MS, Colley RC, Saunders JT, Healy GN, Owen N. Physiological and health implications of a sedentary lifestyle. Appl Physiol Nutr Metab. 2010; 35: 725-40.

4. United States Department of Health and Human Services. Physical activity and health: a report from the Surgeon General. Atlanta: United States Department of Health and Human Services, Center for Disease Control and Prevention, National Center for Chronic Disease Prevention and Health Promotion; 1996.

5. Lee IM, Shiroma EJ, Lobelo F, Puska P, Blair SN, Katzmarzyk PT, et al. Effect of physical inactivity on major non-communicable diseases worldwide: an analysis of burden of disease and life expectancy. Lancet. 2012; 380 (9838), 219-29.

6. Hallal PC, Andersen LB, Bull FC, Guthold R, Haskel W, Ekelund U, et al. Global physical activity levels: surveillance progress, pitfalls, and prospects. Lancet. 2012; 380 (9838): 219-29.

7. Juneau CE, Potvin L. Trends in leisure, transport, and work-related physical activity in Canada 1994-2005. Prev Med. 2010; 51: 384-6.

8. Silva ICM, Knuth AG, Mielke GI, Azevedo MR, Gonçalvez H, Hallal PC. Trends in leisure-time physical activity in a Southern Brazilian city: 2003-2010. J Phys Act Health. 2014. In press

9. Mello MT, Fernandez AC, Tufik S. Levantamento epidemiológico da prática de atividade física na cidade de São Paulo. Rev Bras Med Esporte. 2000; 6(4): 119- 24. 
10. Gómez LF, Mateus JC, Cabrera G. Leisure-time physical activity among women in a neighbourhood in Bogotá, Colombia: prevalence and socio-demographic correlates. Cad Saúde Pública. 2004; 20(4): 1103-9.

11. Florindo AA, Hallal PC, Moura EC, Malta DC. Prática de atividades físicas e fatores associados em adultos, Brasil, 2006. Rev Saude Publ. 2009; 43(2):65-73.

12. Knuth AG, Bacchieri G, Victora CG, Hallal PC. Temporal trends in physical activity: a systematic review. J Phys Act Health. 2009; 6(5): 548-59.

13. VIGITEL Brasil 2012: Vigilância de fatores de risco e proteção para doenças crônicas por inquérito telefônico. Brasília: Ministério da Saúde, Secretaria de Vigilância em Saúde, Secretaria de Gestão Estratégica e Participativa; 2012.

14. Mielke GI, Hallal PC, Malta DC, Lee IM. Time trends of physical activity and television viewing time in Brazil: 2006-2012. Int J Behav Nutr Phys Act. 2014; 15;11:101

15. Izrael D, Hoaglin DC, Battaglia MP. A SAS Macro for Balancing a Weighted Sample. Proceedings of the Twenty-Fifth Annual SAS Users Group International Conference, Paper 275, 2000. [http://www2.sas.com/proceedings/sugi29/207-29.pdf]

16. Bernal RTI, Malta DC, Araújo TS, Silva NN. Telephone survey: post-stratification adjustments to compensate non-coverage bias in city of Rio Branco, Northern Brazil. Rev Saude Publica. 2013,47: 316-25.

17. Mielke GI, Silva IC, Owen N, Hallal PC. Brazilian adults' sedentary behaviors by life domain: population-based study. PlosOne. 2014; 9(3): e91614.

18. Hallal PC, Cordeira K, Knuth AG, Mielke GI, Victora CG. Ten-year trends in total physical activity practice in Brazilian adults: 2002-2012. J Phys Act Health. 2014. In press

19. Matsudo VKR, Matsudo SM, Araújo TL, Andrade DR, Oliveira SC, Hallal PC, et al. Time Trends in Physical Activity in the State of São Paulo, Brazil: 2002-2008. Med Sci Sports Exerc. 2010; 42(12): 2231-6.

20. Viñas BR, Majem LS, Barba LR, Cuspinera ER, Cabezas C, Vallbona C, et al. Trends in physical activity status in Catalonia, Spain (1992-2003). Public Health Nutr. 2010; 10(11A): 1389-95.

21. Nang SW, Howard AG, Wang HJ, Su C, Zhang, B. The physical activity transition among adults in China: 1991-2011. Obes Rev. 2013;1:27-36.

22. Barengo NC, Nissinen A, Tuomilehto J, Pekkarinen. Twenty-five-year trends in physical activity of 30- to 59-year-old populations in eastern Finland. Med Sci Sports Exerc. 2002;02:1302-3408.

23. Silva SG, Duca GF, Silva KS, Oliveira ESA, Nahas MV. Deslocamento para o trabalho e fatores associados em industriários do sul do Brasil. Rev Saude Publ. 2012,46(1):180-4.

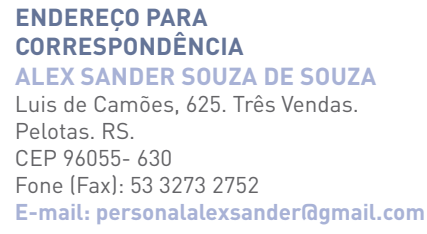

